\title{
Accurate and Precise External Calibration Enhances the Versatility of Quantitative NMR (qNMR)
}

\author{
Yuzo Nishizaki, David C. Lankin, Shao-Nong Chen, and Guido F. Pauli
}

Affiliation Corresponding Author

Guido F. Pauli - Center for Natural Product Technologies (CENAPT), Pharmacognosy Institute (PI), and Department of Pharmaceutical Sciences (PSCI), College of Pharmacy, University of Illinois at Chicago, Chicago, IL 60612, United States; orcid.org/0000-0003-1022-4326; Phone: 1-312-355-1949; Email: gfp@uic.edu

\section{Affiliation Authors}

Yuzo Nishizaki - Pharmacognosy Institute (PI) and Department of Pharmaceutical Sciences (PSCI), College of Pharmacy, University of Illinois at Chicago, Chicago, IL 60612, United States; and Division of Food Additives, National Institute of Health Sciences, Kawasaki-ku, Kawasaki, Kanagawa, 210-9501, Japan; orcid.org/0000-0002-4279-2638

David C. Lankin - Center for Natural Product Technologies (CENAPT), Pharmacognosy Institute (PI), and Department of Pharmaceutical Sciences (PSCI), College of Pharmacy, University of Illinois at Chicago, Chicago, IL 60612, United States; orcid.org/0000-0003-3166-5980

Shao-Nong Chen - Center for Natural Product Technologies (CENAPT), Pharmacognosy Institute (PI), and Department of Pharmaceutical Sciences (PSCI), College of Pharmacy, University of Illinois at Chicago, Chicago, IL 60612, United States; orcid.org/0000-0003-0748-0863

\section{- SUPPORTING INFORMATION}




\section{- TABLE OF CONTENTS}

Figure S1. Relationship of receiver gain $(\mathrm{RG})$ and signal to noise $(\mathrm{S} / \mathrm{N})$ ratio $\quad \mathrm{S}-2$

Figure S2. Non-linear curve fitting for $90^{\circ}$ pulse width (90 PW) determination. S-3

$\begin{array}{lll}\text { Figure S3. } & \text { Quantitative results processed by MestReNova }\end{array}$

Table S1. Inverse correlation between Q factor and $90 \mathrm{PW}$ in a highly conductive sample $\quad$ S-5

Table S2. Heating effects from ${ }^{13} \mathrm{C}$ decoupling on absolute integral values of $\mathrm{DMSO}_{2}$ in sample 12 under different duty cycle times $\quad$ S-6 
Figure S1. Relationship of receiver gain $(\mathrm{RG})$ and signal to noise $(\mathrm{S} / \mathrm{N})$ ratio. This experiment used a sample of $35.3 \mathrm{mM} \mathrm{DMSO}_{2}$ in DMSO- $d_{6}$ in a $5-\mathrm{mm} \mathrm{NMR}$ tube.

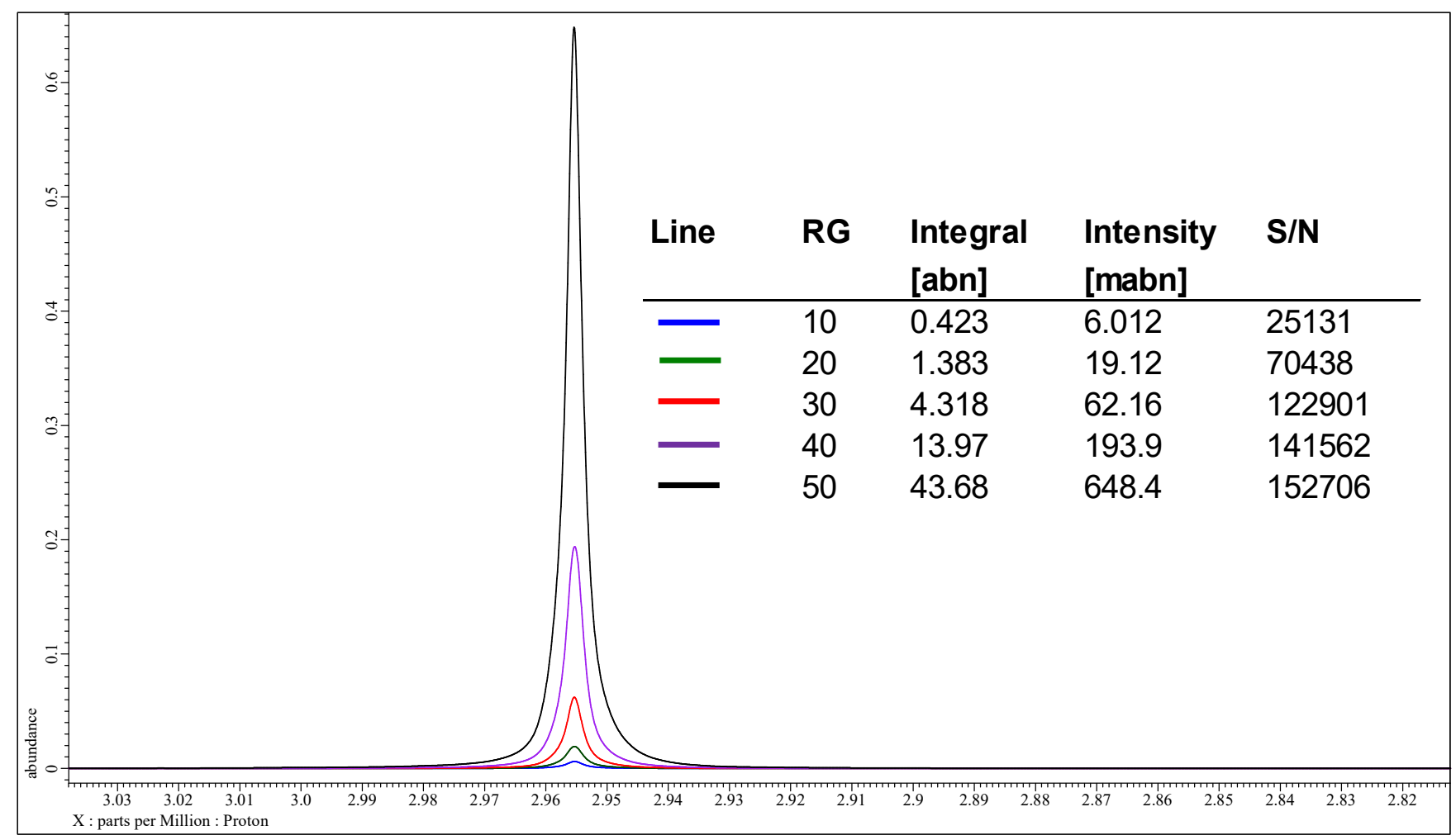


Figure S2. The results of non-linear curve fitting for the determination of the 90 PW using JEOL Delta software. The intensity of the residual solvent signal (DMSO- $d_{5}$ ) acquired on-resonance with varying PWs are plotted. Calculated $90 \mathrm{PWs}$ are displayed in a small box on the bottom right of each screenshot.
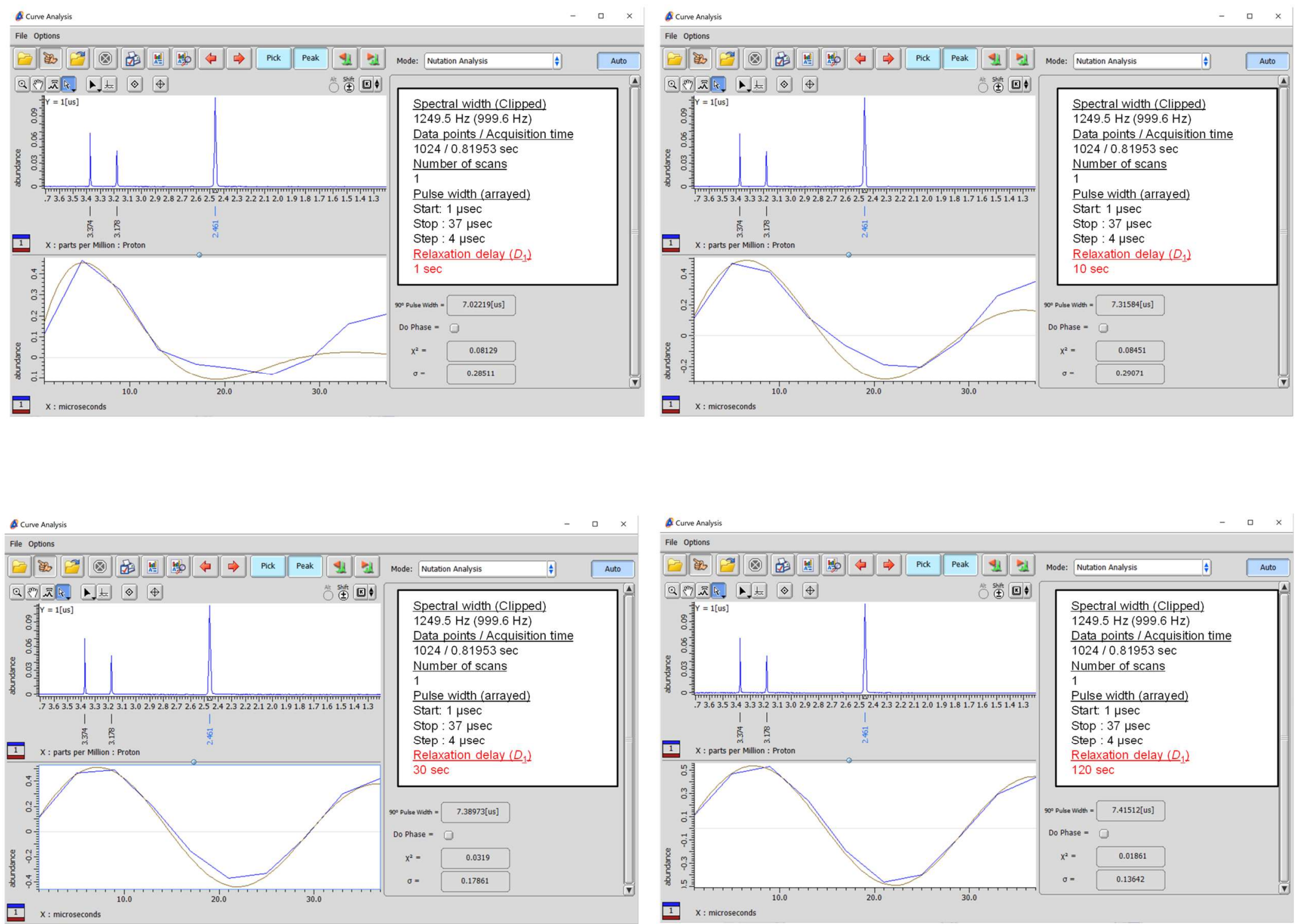
Figure S3. Quantitative results processed by MestReNova. For the comparison, see Figure 2 showing the data obtained from processing with Delta software. The FID was zero filled to 262,144 points (4 times) without any window functions. Then the phase of the spectrum was adjusted manually, and finally the baseline was corrected using cubic splines.

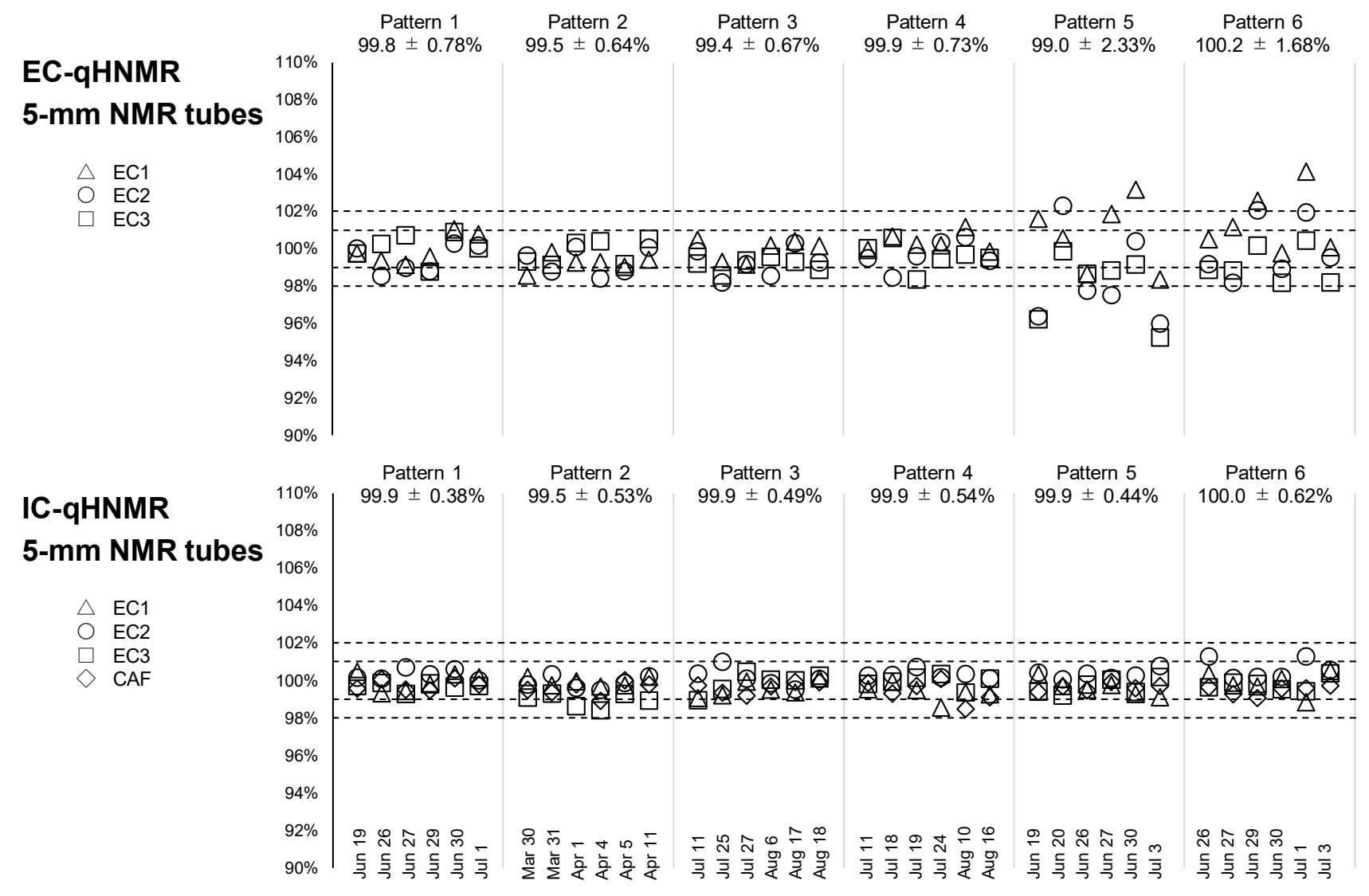


Table S1. The inverse correlation between Q factor and 90 PW in highly conductive samples.

$\begin{array}{lllll} & & \text { Q factor }^{a} & 90 \mathrm{PW}(\mu \mathrm{s}) & \text { Q factor } \times 90 \mathrm{PW} \\ \text { Sample 9 } & n=1 & 21.150 & 7.3102 & 154.61 \\ & n=2 & 21.228 & 7.3051 & 155.08 \\ \text { Sample 10 } & n=3 & 21.451 & 7.3237 & 157.10 \\ & n=1 & 21.199 & 7.3267 & 155.32 \\ & n=3 & 21.417 & 7.3473 & 157.35 \\ \text { Sample 11 } & n=1 & 19.546 & 8.0216 & 156.79 \\ & n=2 & 19.535 & 7.9638 & 155.57 \\ & n=3 & 19.599 & 7.9039 & 154.91\end{array}$

${ }^{a}$ The Q factor represents the integral per proton per molar concentration in 5mm NMR tubes. These values are from EC-qHNMR using pattern 4 in 5-mm NMR tubes with ${ }^{13} \mathrm{C}$ decoupled acquisition. The quantitative values are summarized in Table 3. 
Table S2. The impact of heating effects from ${ }^{13} \mathrm{C}$ decoupling on the absolute integral values of $\mathrm{DMSO}_{2}$ in sample 12 with different duty cycle times.

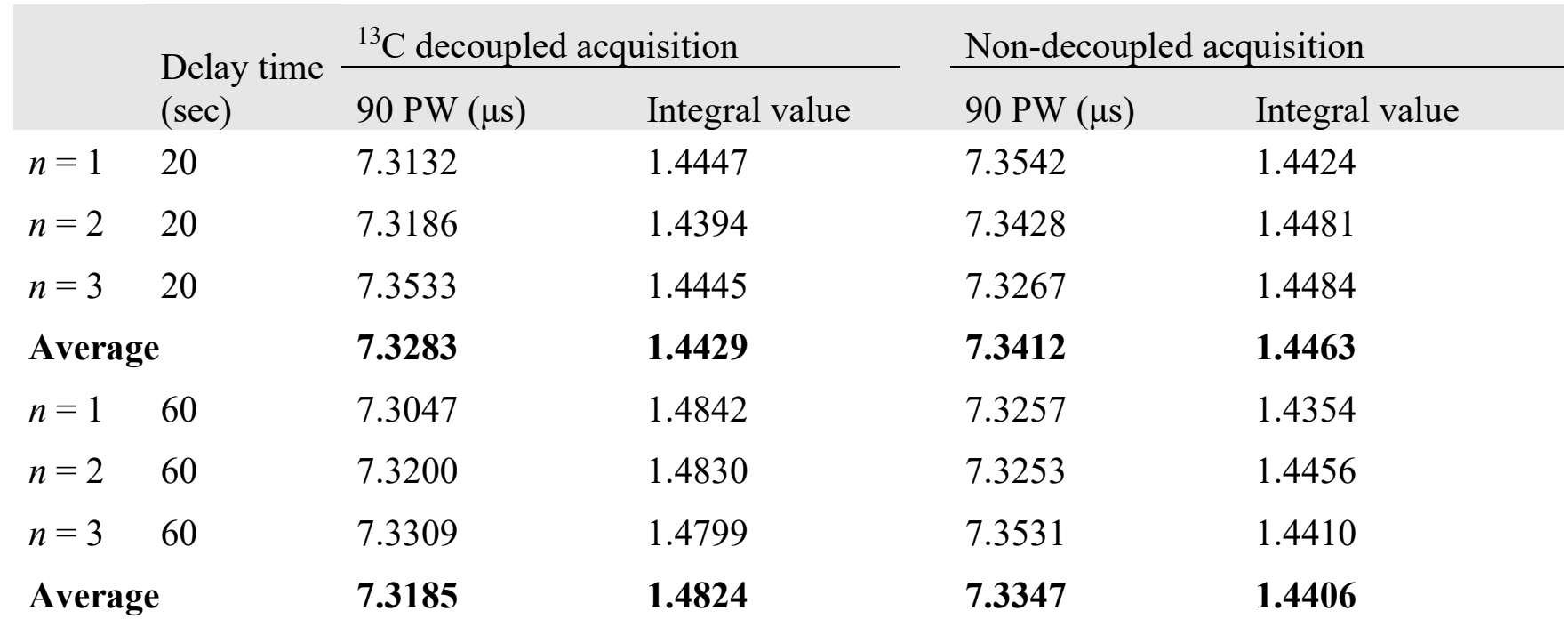

These values are from EC-qHNMR using pattern 4 in 5 -mm NMR tubes with ${ }^{13} \mathrm{C}$ decoupled or nondecoupled acquisition. 